\title{
对位取代四苯基吓啉铜（II）的 生成动力学及其它金属离子的影响
}

\author{
朱志昂* 夏新 陈荣悌 \\ (南开大学 化学系, 天洲 300071)
}

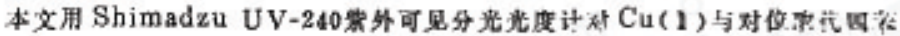

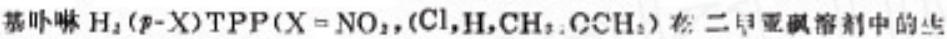

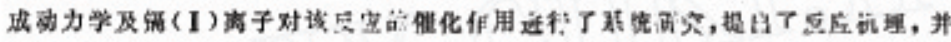

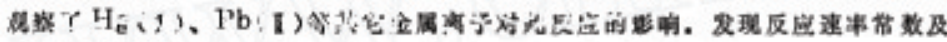

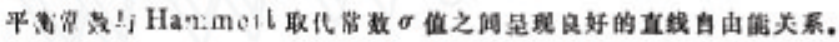

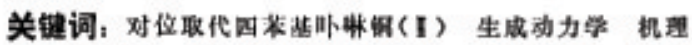

金属吓啉广泛地存在于生物、植物体内, 如血红蛋白、细胞色素 C 及叶绿素等. 金属卟 啉生成动力学是吓啉化学乃至生物体系中的重要课题. 分析化学中, 常用金属吓啉的生成反 应来鉴定或测定微量甚至痕量元素, 也常用销、手等金属离子来促进反应, 但对促进机理研 究甚少. 了解金属吓欶生成反应的机理和条件, 对研究卟啉配合物于生物、植物体内活动机 理均有重要价值.

在前文 ${ }^{[1]}$ 的基础上，本文系统地研究了对位取代四莱基卟啉 $\mathrm{H}_{2}(p-X) \mathrm{TPP} \mathrm{L}_{\mathrm{j}} \mathrm{Cu}$ ( II ) 在 Cd (II) 催化下的生成动少, 求得了动力学参数, 并探讨了它们分别与 Hammett 取 代 常数 的关系. 在观察了温度对 $\mathrm{Cu}\left(\mathrm{p}-\mathrm{OCH}_{3}\right) \mathrm{TPP}$ 生成反应的影响 之后, 求得了反应前置平衡步的

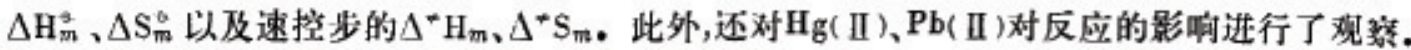

\section{实 验 部 分}

对位取代四策基卟啉 $\mathrm{H}_{2}(p-\mathrm{X}) \mathrm{TPP}$ 按文献 ${ }^{[2,3]}$ 方法合成，其元素分析结果如丧 1 所示.

表1 $\mathbf{H}_{\mathrm{z}}(\boldsymbol{p}-\mathbf{X}) \mathbf{T P P}$ 的元素分析结果

Table 1 Results of the elemental analysis of compounds of $\mathrm{H}_{2}(\mathrm{p}-\mathrm{X}) \mathrm{TPP}$

\begin{tabular}{lrrrrrr}
\hline $\mathrm{X}$ & \multicolumn{2}{c}{$\mathrm{C} \%$} & \multicolumn{2}{c}{$\mathrm{H} \%$} & \multicolumn{2}{c}{ N\% } \\
& Calc. Found & \multicolumn{2}{c}{ Calc. Found } & \multicolumn{2}{c}{ Found } \\
\hline $\mathrm{NO}_{2}$ & 66.50 & 66.52 & 3.30 & 3.41 & 14.10 & 14.18 \\
$\mathrm{Cl}$ & 70.23 & 70.09 & 3.48 & 3.29 & 7.45 & 7.23 \\
$\mathrm{H}$ & 85.97 & 84.93 & 4.92 & 4.62 & 9.11 & 8.97 \\
$\mathrm{CHa}$ & 85.94 & 85.61 & 5.71 & 5.71 & 8.35 & 7.97 \\
$\mathrm{OCH}_{3}$ & 78.46 & 75.37 & 5.21 & 5.27 & 7.62 & 6.78 \\
\hline
\end{tabular}

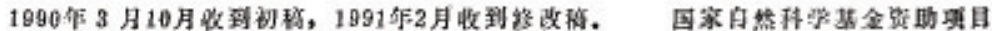


所用无水盐 $\mathrm{Cd}(\mathrm{OAc})_{2} 、 \mathrm{CuCl}_{2} 、 \mathrm{KNO}_{3}$ 及溶剂二甲亚砜的处理和储存方法同文献 $[1]$.

本文使用的是 Shimadzu UV- 240 紫外可见分光光度计, 温度控制使用 Shimadzu TB85佰 温槽, 精度 $0.1^{\circ} \mathrm{C}$. 反应在金属离子浓度大大过量于 $\mathrm{H}_{2}(p-\mathrm{X}) \mathrm{TPP}$ 浓度的假一级条件下进行. 在特征波长 $514.5 \mathrm{~nm}$ 下测量反应体系吸光度随时间变化值, 用Guggenheim 方法 ${ }^{[4]}$ 求得反应的 表观速率常数 $k_{\mathrm{obs}}$, 再用非线性阻尼最小二乘法对实验点进行拟合, 求得前置平衡常数及 速控步的速率常数.

\section{实验结果和讨论}

1. 反应机理在 $50^{\circ} \mathrm{C} 、 \mathrm{Cu}($ II $)$ 离子旅度 $\left(\sim 10^{-2} \mathrm{~mol}^{\circ} \cdot \mathrm{dm}^{-3}\right)$ 一定的条件下, 改变 $\mathrm{Cd}$ (II) 离子旅度 $\left(0 \sim 10^{-2} \mathrm{~mol} \cdot \mathrm{dm}^{-3}\right)$, 测量 $\mathrm{Cu}(p-\mathrm{X}) \mathrm{TPP}$ 生成反应的㖟光度随时间的变 化, 求得表 观速事常数 $k_{\mathrm{obs}}$ 与 $\mathrm{Cd}$ (II) 离子浓度的关系, 如图 1 中的点所示. 从图可以看出, 在 $[\mathrm{Cd}$ (II)]

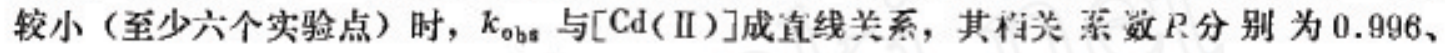

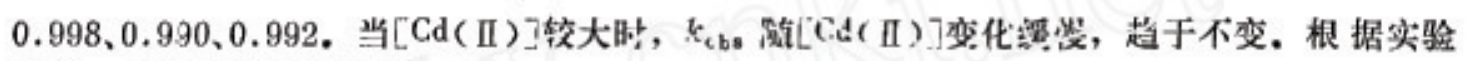

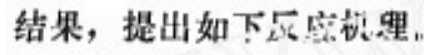

$\mathrm{Cu}$ (II) 与 $\mathrm{K}_{2}(p \cdots \mathrm{X}) \mathrm{TPF}$ 生成反这的机理 (机理 I) 为 ${ }^{[1]}$,

$$
\begin{array}{r}
\mathrm{Cu}(\text { II })+\mathrm{H}_{2}(p-\mathrm{X}) \mathrm{TPP} \stackrel{\mathrm{K}}{\rightleftharpoons} \mathrm{Cu}^{*} \mathrm{H}_{2}(p-\mathrm{X}) \mathrm{TPP} \\
\mathrm{C}{ }^{*} \mathrm{H}_{2}(p-\mathrm{X}) \mathrm{TPP} \stackrel{k}{\longrightarrow} \mathrm{Cu}(p-\mathrm{X}) \mathrm{TPP}+2 \mathrm{H}^{+}
\end{array}
$$

此机理的表观速率常数可表示为;

$$
k_{\text {obs }}=k \cdot \mathrm{K}[\mathrm{Cu}(\text { II })] /(1+\mathrm{K}[\mathrm{Cu} \text { ( II ) }])
$$

有 $\mathrm{Cd}$ ( II ) 离子存在时, 其反应机理 (机理 II) 为:

$$
\begin{gathered}
\mathrm{Cd}(\text { II })+\mathrm{H}_{2}(p-\mathrm{X}) \mathrm{TPP} \stackrel{K_{1}}{\rightleftharpoons} \mathrm{Cd}^{*} \mathrm{H}_{2}(p-\mathrm{X}) \mathrm{TPP} \\
\mathrm{Cd}^{*} \mathrm{H}_{2}(p-\mathrm{X}) \mathrm{TPP}+\mathrm{Cu}(\text { II }) \stackrel{k_{1}}{\longrightarrow} \mathrm{Cu}(p-\mathrm{X}) \mathrm{TPP}+\mathrm{Cd}(\text { II })+2 \mathrm{H}^{+} \\
\mathrm{Cu}(\text { II })+\mathrm{H}_{2}(p-\mathrm{X}) \mathrm{TPP} \stackrel{K_{2}}{\rightleftharpoons} \mathrm{Cu}^{*} \mathrm{H}_{2}(p-\mathrm{X}) \mathrm{TPP} \\
\mathrm{Cu} * \mathrm{H}_{2}(p-\mathrm{X}) \mathrm{TPP} \stackrel{k_{2}}{\longrightarrow} \mathrm{Cu}(p-\mathrm{X}) \mathrm{TPP}+2 \mathrm{H}^{+} \\
k_{\text {ots }}=\left(k_{1} K_{1}[\mathrm{Cd}(\text { II })]+k_{2} K_{2}\right)[\mathrm{Cu}(\text { II })] /\left(1+K_{1}[\mathrm{Cd}(\text { II })]+K_{2}[\mathrm{Cd}(\text { II })]\right)
\end{gathered}
$$

其中 $K 、 K_{1} 、 K_{2}$ 为前置平衡步的平衡常数, $k_{1} k_{1} 、 k_{2}$ 为速控步的速率常数.

当 $[\mathrm{Cd}($ II $)]=0$ 时, 机理 II 还原为机理 I, (8) 式还原为 (3) 式. 根据 (8) 式, 用非线性阻尼 最小二乘法对图 1 中的各实验点进行拟合, 同时求 得机理 II 中的各动力学数据和拟合的相对误差平方 和 $S$, 列于衣 2 . 图 1 中的曲线即为拟合曲线, 其 与实验点很好地吻合, 说明了提出机理的合理性. 此外, 从( 8 )式可以看出, 在 $[\mathrm{Cu}$ (II)]值( $\left.10^{-2} \mathrm{~mol} \cdot \mathrm{dm}^{-3}\right)$ 一定, [Cd(II)]较低 时, (8)式可 变为:

$$
\begin{aligned}
k_{\mathrm{obs}}= & k_{1} K_{1}[\mathrm{Cd}(\text { II })][\mathrm{Cu}(\text { II })] /\left(\mathrm{I}+K_{2}[\mathrm{Cu}(\text { II })]\right. \\
& +k_{2} K_{2}[\mathrm{Cu}(\text { II })] /\left(1+K_{2}[\mathrm{Cu}(\text { II })] \quad\right. \text { ( 9 ) }
\end{aligned}
$$

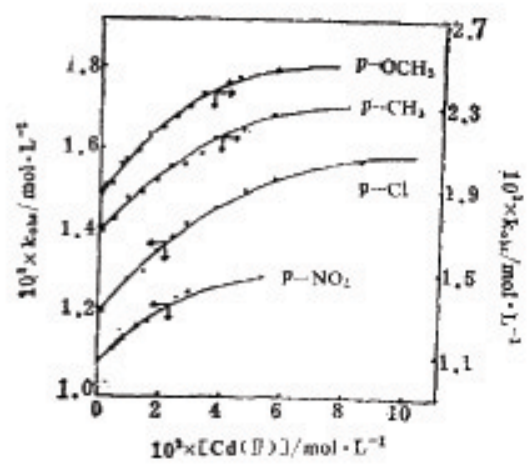

图 $150^{\circ} \mathrm{C} \mathrm{H}(p-\mathrm{X}) \mathrm{TPP} 5 \mathrm{Cu}(\mathrm{I})$ 反底 的 $k_{\mathrm{ob}} \sim[\mathrm{Cd}(1)]$ 美菜图

Fig.1. Plot of $k_{o b s}$ vs. $[\mathrm{Cd}(\mathrm{I})]$ of $\mathrm{H}_{2}(p-\mathrm{X}) \mathrm{TPP}$ at $50^{\circ} \mathrm{C}$ 
此时, $k_{\mathrm{obs}}$ 与 $[\mathrm{Cd}$ (II) $]$ 成直线关系, 这与实验结果是一致的. 在 $[\mathrm{Cd}(\mathrm{II})]$ 旅度 较大时, 且 $K_{1}[\mathrm{Cd}$ (II) $] \gg K_{2}[\mathrm{Cu}$ (II) $]$ 时, 从 ( 8 ) 式看出, $k_{o b s}$ 应与 $[\mathrm{Cd}($ II $)]$ 无关, 应为常数, 实 验 中 已看出曲线趋于水平的趋势.

絜2 50 ○时 $\mathrm{Cu}(\boldsymbol{l})$ 与 $\mathrm{H}_{2}(\boldsymbol{p}-\mathrm{X}) \mathrm{TPP}$ 反应的动力学数据

Table 2 The kinetic data of reaction of $\mathrm{Cu}(\mathrm{I})$ with $\mathrm{H}_{2}(p-\mathrm{X}) \mathrm{TPP}$ at $50^{\circ} \mathrm{C}$

\begin{tabular}{|c|c|c|c|c|c|c|}
\hline $\mathrm{x}$ & $K_{1} /\left(\mathrm{mol}^{-1} \cdot \mathrm{dm}^{2}\right)$ & $K_{\mathrm{s}} /\left(\mathrm{mol}^{-1} \cdot \mathrm{dm}^{2}\right)$ & $k_{1} /\left(s^{-2}\right)$ & $k_{2} /\left(s^{-1}\right)$ & $S /\left(10^{-4}\right)$ & Notes \\
\hline $\mathrm{NO}_{2}$ & 202.8 & 10.52 & 0.1046 & 0.006925 & 3.7 & This work \\
\hline $\mathrm{Cl}$ & 174.6 & 12.55 & $0.146 \$$ & 0.008575 & 6.7 & This work \\
\hline II & 232.3 & 12.35 & 0.1617 & 0.008849 & 4.5 & $\mathrm{ref} \cdot[1]$ \\
\hline r.f: & 243.2 & 24.62 & 0.2082 & 0.01056 & 13 & This work \\
\hline$O \mathrm{CH}_{3}$ & 263.9 & 18,54 & 0.5591 & $0.0 ! 401$ & 7.4 & TH 3 work \\
\hline
\end{tabular}

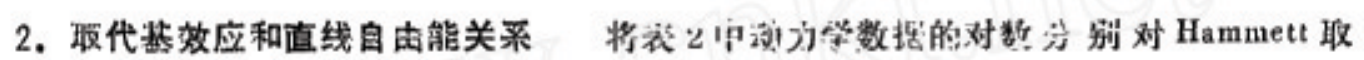

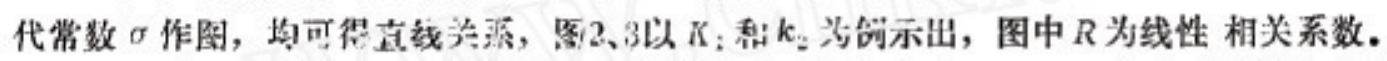

图2、3中直线皆以 $\mathrm{X}=\mathrm{H}$ 为折点, 这说明给电子取优基 $\left(\mathrm{OCH}_{3}, \mathrm{CH}_{3}\right)$ 和吸电子取代基 $\left(\mathrm{NO}_{2}, \mathrm{Cl}\right)$ 对反应的影唎不同. 给电子取代基使 $\mathrm{H}_{2}(p-\mathrm{X}) \mathrm{TPP}$ 的 $\mathrm{N}$ 上电子云密度 增大, 有 利子 $\mathrm{Cu}$ (II) 离子的珫入而生成 $\mathrm{Cu}(p-\mathrm{X}) \mathrm{TPP}$. 从表 2 可看出, 取代基给电子能力食大, 则 $K_{1} 、 K_{2}, k_{1} 、 k_{2}$ 也急大, 而吸电子取代基的影响㭘好相反.

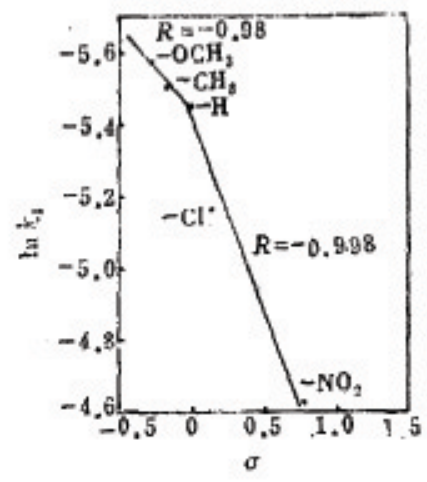

图 $2 \ln K_{1} \sim \sigma$ 图

Fig.2 Plot of $\ln K_{\text {, vs. } \sigma}$

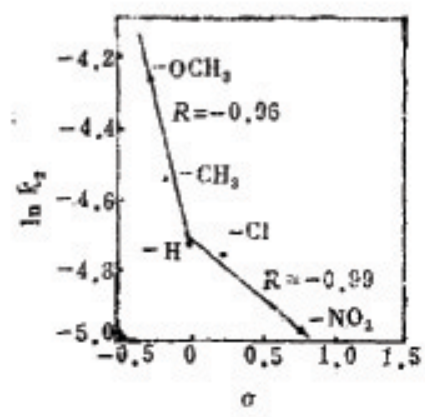

圈 $3 \ln k_{2} \sim U$ 图

Fig.3 Plot of $\ln k_{3}$ v $s, \sigma$

根据表 2 中数据, 发现如下直线自由能关系:

对给电子取代基:

$$
\begin{aligned}
& \ln K_{1}=3.8 \ln K_{2}-4.3 \\
& \ln k_{1}=0.548 \ln K_{1}-4.78 \\
& \ln k_{2}=0.31 \ln K_{1}-6.4 \\
& \ln k_{1}=2.2 \ln K_{2}-7.4 \\
& \ln k_{2}=1.30 \ln K_{2}-8.03 \\
& \ln k_{2}=0.580 \ln k_{1}-3.69
\end{aligned}
$$

$$
\begin{aligned}
& R=0.94 \\
& R=0.990 \\
& R=0.97 \\
& R=0.98 \\
& R=0.993 \\
& R=0.996
\end{aligned}
$$


对吸电子取代基：

$$
\begin{array}{ll}
\ln K_{1}=0.305 \ln K_{2}+4.68 & R=0.998 \\
\ln k_{1}=6.69 \ln K_{1}-38.3 & R=0.990 \\
\ln k_{2}=3.84 \ln K_{1}-25.6 & R=0.997 \\
\ln k_{1}=2.06 \ln K_{2}-7.07 & R=0.996 \\
\ln k_{2}=1.1770 \ln K_{2}-7.7060 & R=0.99998 \\
\ln k_{2}=0.557 \ln k_{1}-3.68 & R=0.997
\end{array}
$$

\begin{tabular}{|c|c|c|c|c|c|}
\hline$t /{ }^{\circ} \mathrm{C}$ & $K_{1} /\left(\mathrm{mol}^{-1} \cdot \mathrm{dm}^{3}\right)$ & $K_{\mathrm{a}} /\left(\mathrm{mol}^{-1} \cdot \mathrm{dm}^{3}\right)$ & $k_{1} /\left(s^{-1}\right)$ & $k_{2} /\left(s^{-1}\right)$ & $S /\left(10^{-4}\right)$ \\
\hline 35 & 123.3 & 10.72 & 0.09963 & 0.005593 & 8.9 \\
\hline 40 & 181.4 & 13.28 & 0.1541 & 0.008030 & 6.0 \\
\hline 45 & 249.8 & 16.01 & 0.2148 & 0.01055 & 3.0 \\
\hline 50 & 262.0 & 18.55 & 0.3591 & 0.01401 & 7.4 \\
\hline$\Delta H_{m}^{*} / \mathrm{k} f \cdot \mathrm{mol}^{-1}$ & 42.80 & 30.31 & & & \\
\hline \multirow{2}{*}{$\Delta s_{m}^{a} / \sqrt{ } \operatorname{lnol}^{-1} \cdot \mathrm{K}^{-1}$} & 179.5 & 118.2 & & & \\
\hline & $R=0.96$ & $R \propto 0.998$ & & & \\
\hline$\Delta+\mathrm{H}_{m} / \mathrm{kJ} \cdot \mathrm{mol}^{-1}$ & & & 66.44 & 44.02 & \\
\hline \multirow[t]{2}{*}{$\Delta+\mathrm{S}_{\mathrm{m}} / \mathrm{J} \cdot \mathrm{mol}^{-1} \cdot \mathrm{K}^{-1}$} & & & -48.95 & -144.9 & \\
\hline & & & $R=0.996$ & $R=0.99998$ & \\
\hline
\end{tabular}

这些直线关系，与上述取代基效应的直线关系相同，仍是以 $\mathrm{X}=\mathrm{H}$ 为折点的.

3. 湿度影响在 $\mathrm{Cu}$ (II) 离子浓度一定, 不同 $\mathrm{Cd}$ (II)离子浓度条 件下, 在不同温度 下, 测量 $\mathrm{H}_{2}\left(p-\mathrm{OCH}_{3}\right) \mathrm{TPP}$ 与 $\mathrm{Cu}$ (II) 生成 $\mathrm{Cu}\left(p-\mathrm{OCH}_{3}\right) \mathrm{TPP}$ 反应的 $k_{\mathrm{obs}}$, 根据机理 II, 对实 验数据进行拟合, 并求得不同温度时的动力学参数 $K_{1} 、 K_{2}, k_{1} 、 k_{2}$, 如表 3 所示.

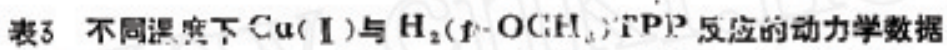

Table 3 The kigene data of reaction of $\mathrm{Cs}(5)$ with $\mathrm{H}_{2}\left(p-\mathrm{OCH}_{3}\right) \mathrm{TPP}$ at different temperature

根据动力学 Eyring 公式, 以 $\ln \left(k_{1} / T\right)$ 对 $1 / T$ 线性回归, 求得速控步的活化站 $\Delta^{*} H_{\mathrm{m}, 1}$, 活化嫡 $\Delta^{*} S_{m, 1}$, 同理以 $\ln \left(k_{2} / T\right)$ 对 $1 / T$ 线性回归, 求得相应的 $\Delta^{*} H_{m, 2}, \Delta^{*} S_{m, 2}$; 再 根 据 $\Delta G_{m}^{*}=-R T \ln K=\Delta H_{m}^{\circ}-T \Delta S_{m}^{\circ}$, 以 $\ln K$ 对 $1 / T$ 线性回归, 分别求得前犆平衡步的标准摩 尔焓 $\Delta H_{m, 1}^{\circ}, \Delta H_{m, 2}^{\circ}$, 标准摩尔桷 $\Delta S_{m, 1}^{\mathrm{a}}, \Delta S_{m, 2}^{\circ}$, 其数值及线栍相关系数亦列于表 3 中.

从表 3 中数据可以看出, 随温度的升高, 反应各步的动力学常数是增加的, 即反应是加 快的. 从 $35^{\circ} \mathrm{C}$ 升高到 $50^{\circ} \mathrm{C}, k_{1}$ 增加3.6倍, 而 $k_{2}$ 增加 2.3 倍, 这是由于 $\Delta^{*} H_{m, 1}$ 稍大于 $\Delta^{*} H_{m, 2}$ 之故. 同时, $\Delta^{*} H_{m, 1}$ 和 $\Delta^{\star} H_{m, 2}$ 为同一数量级, 说明这两步足同时平行进行的竞 争步繁. $\Delta^{*} S_{m, 1}, \Delta^{*} S_{m, 2}$ 为负值, 说明速控步的活化过程是㛙降低过程, 这可认为是由于洛剂化喻的 贡献所致. 在包含带电粒子的溶液中, 溶刘化效应经常支配着活化摘 ${ }^{[5]}$. 速 控 步的活化过 程是吓啉上的氢原子溷步离子化的过程, 二甲亚砳溶剂分子能与离子化的氢原子很好地结 合 ${ }^{[\theta]}$, 使溶剂分子更有序地排列, 从而使膟降低. 而前罚平衛步的 $\Delta S_{m, 1}^{a}, \Delta S_{m, 2}^{e}$ 均为正值,

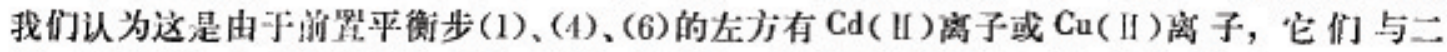

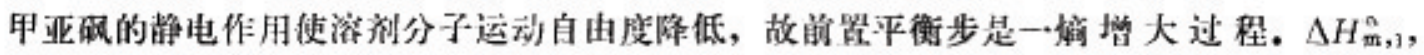

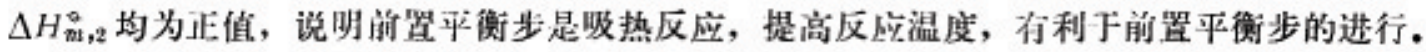


在机理中, 我们认为吓啉上的质子是在速控步(2)、(5)、(7)解离下来的, 这是否正确? 我们可以从下面的假设中找到答案. 若假设吓啉上的质子在前置平衡步解离下来, 此时, 机 理 I 应写成如下形式:

$$
\begin{gathered}
\mathrm{Cu}(\text { II })+\mathrm{H}_{2}(p-\mathrm{X}) \mathrm{TPP} \stackrel{{ }^{K}}{\rightleftharpoons} \mathrm{Cu}^{*}(p-\mathrm{X}) \mathrm{TPP}+2 \mathrm{H}^{+} \\
\mathrm{Cu}^{*}(p-\mathrm{X}) \mathrm{TPP} \stackrel{\boldsymbol{k}}{\longrightarrow} \mathrm{Cu}(p-\mathrm{X}) \mathrm{TPP}
\end{gathered}
$$

据此机理推出的速率方程为:

$$
-\mathrm{d}\left[\mathrm{H}_{2}(p-\mathrm{X}) \mathrm{TPP}\right] / \mathrm{d} t=k K[\mathrm{Cu}(\text { II })]\left[\mathrm{H}_{2}(p-\mathrm{X}) \mathrm{TPP}\right] /\left(\left[\mathrm{H}^{+}\right]^{2}+K[\mathrm{Cu}(\text { II })]\right)
$$

实验中, 采用金属离子大大过量的条件, 可以近似认为金属离子的浓度在反应中不发生 变化, 在这一近似条件下, (3) 式所表示的 $k_{\mathrm{obs}}$ 对一特定反应而㞩为一常数, 即可认为反应 是假一级反应. 而 (12) 式则不同, 由于反应 过程 中 $\mathrm{H}^{+}$不断生 成, $k K\left[\mathrm{Cu}(\mathbb{I}] /,\left(\left[\mathrm{H}^{+}\right]^{2}+\right.\right.$

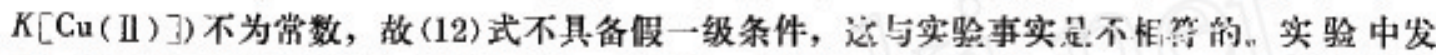

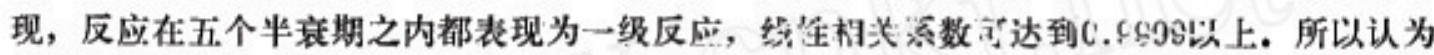
吓濑上的质子在速控㠿解要起保理的。

\section{4. 金属禹字经的化作用}

(1) $\mathrm{Cd}$ ( II) 离子的影响 实验得到 Cd ( II) 离子明显加速 $\mathrm{H}_{2}(p-\mathrm{X}) \mathrm{TPP}$ 与 $\mathrm{Cu}$ ( II ) 晌反 应. 这是由于, 根据我们提出的反应机理 II, 当有 $\mathrm{Cd}($ II ) 离子存 在 时, $\mathrm{Cu}(p-\mathrm{X}) \mathrm{TPP}$ 的生 成有两种同时进行的途径, 与机理 I 相比, 增加了一条途径. 在机理 II 中, $\mathrm{Cd}$ ( II) 可以先与 $\mathrm{H}_{2}(p-\mathrm{X}) \mathrm{TPP}$ 生成 $\mathrm{Cd}^{*} \mathrm{H}_{2}(p-\mathrm{X}) \mathrm{TPP}$, 而后被 $\mathrm{Cu}$ (II) 离子进攻, 生成产 物 $\mathrm{Cu}(p-\mathrm{X}) \mathrm{TPP}$. 从 表 3 数据可以看出, $k_{1}$ 比 $k_{2} 、 K_{1}$ 比 $K_{2}$ 都大一个数量级, 可见由 $\mathrm{Cd}($ II ) 漓子 参 与的途径更 易进行, 从而明显加速反应.

(2) $\mathrm{Pb}$ (II) $、 \mathrm{Hg}$ (II) 离子的影响 在 $35^{\circ} \mathrm{C} 、[\mathrm{Cu}$ (II) $]=0.009328 \mathrm{~mol} . \mathrm{dm}^{-3}$ 付, 我们 分别观察了有 $\mathrm{Cd}\left(\right.$ II ) $、 \mathrm{~Pb}$ (II) 、 $\mathrm{Hg}$ (II) 存在时, CuTPP 的生成情况, 并求出了 $k_{\text {obs }}$, 发 现 这三种金属离子对 CuTPP 的生成都有促进作用，由表 4 可见，有这三种金属离子存在时的 $k_{\mathrm{oba}}$ 都比无其它离子时的大, 表中 $R$ 为假一级反应的线性相关系数.

$\mathrm{Cd}$ ( II )、 $\mathrm{Pb}$ ( II )、 $\mathrm{Hg}$ ( II ) 加速反应的原因是相似的. 这三种金属离子的离子半径都较大， 使得它们与 $\mathrm{H}_{2} \mathrm{TPP}$ 生成的 $\mathrm{M}^{*} \mathrm{H}_{2} T P P(\mathrm{M}=\mathrm{Cd}(\mathrm{II}) 、 \mathrm{~Pb}$ (II) $、 \mathrm{Hg}$ (II) 是金属离子出出吓㗎平面 的锥形结构, 这种结构中的卟啉发生一定的变形, 建利于 $\mathrm{Cu}$ ( I I ) 病子的进攻 ${ }^{\left[{ }^{7}\right]}$, 从而 起到 加速 CuTPP 生成的作用.

表4 $35^{\circ} \mathrm{C} \mathrm{Cd}\left(\right.$ I) $、 \mathrm{~Pb}$ (I)、 $\mathrm{Hg}$ ( I ) 对 $k_{\mathrm{ob} 8}$ 的影晌

Table 4 The effects of $\mathrm{Cd}(\mathbf{I}), \mathrm{Pb}(\mathbf{I}), \mathrm{Hg}(\mathbf{I})$ on $\boldsymbol{k}_{0 \mathrm{~b}}$ at $35^{\circ} \mathrm{C}$

\begin{tabular}{lllll}
\hline \multicolumn{1}{c}{ Motal lon } & None & $\mathrm{Cd}(\mathrm{I})$ & $\mathrm{Pb}(\mathrm{I})$ & $\mathrm{Hg}(\mathrm{I})$ \\
\hline Conc. $/\left(10^{-1} \mathrm{~mol} \cdot \mathrm{dm}^{-3}\right)$ & & 4.897 & 4.034 & 4.171 \\
$k_{\text {obs }} /\left(10^{-4} \mathrm{~s}^{-1}\right)$ & 2.59 & 3.22 & 3.29 & 3.34 \\
Correlation coofficient & 0.99991 & 0.9998 & 0.9994 & 0.9996 \\
\hline
\end{tabular}




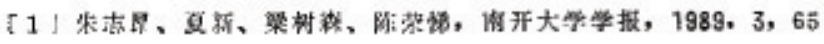

[2] Adler, A.D., J. Org. Chem., 1967, 32, 476

[3] Adlet, A.D., Longo, F.R., Kampas, F., Kim, J., J. Inorg. Nuel. Chem.,1970, 32, 2443

[4] Moore, J.W., Pearson, R.G., "Kinetics and Mechanism", Third Edilion, A WILEY-IN'T-

ERSCIENCE PUBLICATION, New York, p71, 1981

[5] Pearson, R.G., J. Chen. Phys., 1952, 20, 1478

[6] Lau, Y.K., Saluja, P.P.S., Kebarle, P., J. An. Chem. Soc., 1980, 102, 7429

[7] Tanaka, M., Pure \& Appl. Chem., 1983, 55, 151

\title{
KINETICS OF THE FORMATION OF (PAR A-SU QSTITUTED \\ TETRAPHENYLFORP'HINATO; - COPPLR (II) AND \\ THE CATALYTIC EFFECT OF OTHER \\ MATAL IONS
}

\author{
Zhu Zhiang* Xia Xin Chen Rongti \\ (Department of Chemistry, Nankai University, Tianjin 300071)
}

\begin{abstract}
The kinetics of formation reaction of Copper(II) with para-substituted tetraphenylporphyrin $\mathrm{H}_{2}(p-\mathrm{X})$ TPP ( $\mathrm{X}=\mathrm{NO}_{2}, \mathrm{Cl}, \mathrm{H}, \mathrm{CH}_{3}, \mathrm{OCH}_{3}$ ) in dimethyl sulfoxide solution and the catalytic effect of Cadmium (II) ion have been studied on Shimadru UV-240 spectrophotometer. The mechanisms were proposed and the effect of $\mathrm{Hg}$ (II), $\mathrm{Pb}$ (II) on this reaction was investigated. Good LFER (linear free energy relationahips) between reaction rate constant/equilibrium constant with the Hammett subtituent constant $\sigma$ have been ob served in certain cases.
\end{abstract}

Keyword; (Para-substituted tetraphenylporphinato)-Copper(II), Kinetics of formation Mechanisms 